\title{
Analysing the effect of increasing renewable capacities in Great Britain on the regional allocation and wholesale prices
}

\author{
Sara Lupo ${ }^{1 凶}$, Manuel Ruppert ${ }^{2}$, Viktor Slednev ${ }^{2}$, Aristides E. Kiprakis ${ }^{1}$ \\ ${ }^{1}$ Institute for Energy Systems, School of Engineering, The University of Edinburgh, Edinburgh, UK \\ ${ }^{2}$ Chair of Energy Economics, Institute for Industrial Production (IIP), Karlsruhe Institute of Technology \\ (KIT), Karlsruhe, Germany \\ 凶-mail: s.lupo@ed.ac.uk
}

\begin{abstract}
Great Britain has begun to implement significant modifications that are ought to change the national electricity generation. Adding a large amount of renewable capacity to the energy mix will impact different areas of energy use from the landscape used for installation to the electricity market. This study presents an approach to determine the optimal locations of renewable energy installation based on predictions of renewable expansion under consideration of grid constraints. The occurring cost are analysed with regard to the expansion cost and the impact on the wholesale electricity prices.
\end{abstract}

\section{Introduction}

Great Britain is in the process of transforming the way its energy demands are met by shifting from conventional generation by accommodating the energy system to be more suitable for low-carbon energy technologies; however, few steps have been made to ease this transition.

The disconnection within the grid grows further as with the prospective increasing share of renewable generation in the British energy system. This is because more and more decentralised generation is connected below the transmission grid level, yet the distribution grids have not been brought up to date to the needed extent, especially given the fluctuating nature of renewables.

Existing literature, such as [1], has already highlighted the impact of renewable energy sources (RES) expansion in future scenarios on the power system of Great Britain. Focusing on a small-scale geographical horizon, wind and solar generation have been compared in [2] with regard to their suitability for installation. No existing approach, however, combines a larger geographical horizon expansion planning for all major types of RES. With our modelling approach, we analyse the impact of different penetrations of renewable energy generation capacities and growth in electricity including the topology and restrictions from the transmission and distribution grid by applying a cost-minimising renewable expansion planning approach. Additionally, regionalised demand patterns are utilised, which are successively assigned to substations at the voltage level of $132 \mathrm{kV}$ and below.

Since 2011, National Grid has released its predictions for the development of the British energy platform, in the form of the future energy scenarios, [3]. There are four scenarios differing in generating technology capacities based on the green ambition. The gone green scenario is the only one that meets the renewable energy target for the United Kingdom and focuses on fostering renewable energy development. The consumer power scenario focuses more on ensuring energy security within the energy system and reducing generation costs, and so clean technology takes a secondary position. The slow progression scenario makes some progress towards reaching the British green ambition, but less low-carbon capacity is built overall. Finally, the no progression scenario assumes there has been no progress in energy build from the current state.
The impact of different National Grid scenarios is evaluated by comparing both the cost resulting in the required RES expansion as well as the effect of the resulting generation patterns on the wholesale electricity prices.

Great Britain's green goals still abide by its commitments made to the European Union (EU) in the past and despite recent political changes based on the country's decision to leave the EU, the current government maintains it is committed to achieving these goals due to their global, not just European importance, as COP21 proved.

The way forward, as Fig. 1 demonstrates, heavily focuses on onshore, and later, increased offshore wind generation. In January 2017, offshore wind capacity was 5097.6 MW and onshore wind capacity amounted to $9387.65 \mathrm{MW}$, resulting in a combined wind capacity of nearly $14.5 \mathrm{GW}$, [4].

According to the most optimistic gone green scenario, used among others to obtain our results, wind generation is the biggest renewable technology in Britain now and in the future. Cuts by the previous conservative government were made to onshore wind generation; however, National Grid (NG) does not view these as an obstruction to wind energy growth. In terms of capacity, solar photovoltaic (PV) generation rose in numbers every year since 2006, when first panels began to be installed. Cuts to solar subsidies were also made in the summer of 2015 , and a decrease in installations has been observed, but similarly to wind installation, NG does not view this as long-term problem when it comes to future capacity increase.

Biomass generation is not expected to reach numbers similar to wind and solar, however, it is important to note its role, in Great Britain's energy transition, given that the former largest coal-fired power plant Drax is undergoing conversion into biomass firing.

\section{Methodology}

\subsection{Determination of spatial distribution of renewable generation expansion and demand}

2.1.1 Renewable generation expansion: The growth of renewable capacity was largely achieved with the use of time-coupled cost minimisation generation expansion optimisation 


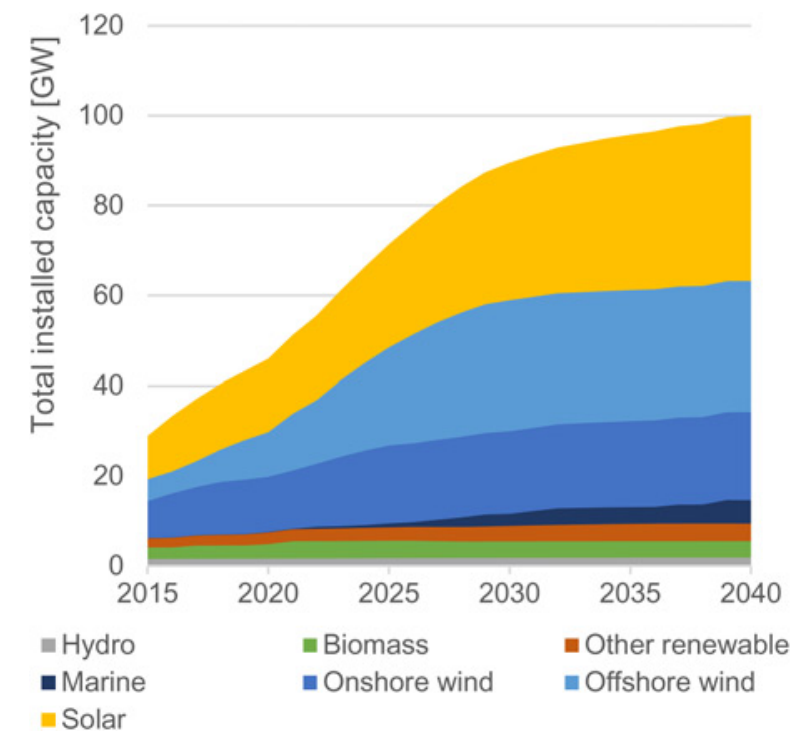

Fig. 1 Capacity development in the gone green scenario [3]

model spanning over the entire time horizon from 2016 to 2040 . The input sources for the renewable expansion model are broken down by type and subtype in Table 1 .

The optimisation variables included the existing set of individual renewable generators down to their specific characteristics, planned investments, greenfield and brownfield potentials, which were defined based on polygons with equal conditions concerning the resource availability and suitability for specific renewable technology options in a given time frame.

Generation technologies included in the modelling approach, which is demonstrated in Fig. 2, are linked both on an inter-technological, as well as on an intra-technological level. As model decisions on investment and divestment of all included technology types have a direct impact on the exploitation of available regional potentials, it means that these decisions are influenced by each other. Furthermore, the capacity restriction imposed by the transmission grid substations limits the total available spatial capacity potential; hence, interlinking the investment and divestment options over all technologies. Finally, scenario assumptions defining regional and national targets, caps and floor for the generation and capacities of RES technologies are added to the constraint matrix.

The objective function is defined as a minimisation of the discounted investment and variable cost with a differentiation of cost based on the planning state of planned investment options and different greenfield and brownfield investment cost. By dividing the objective cost coefficient of each variable by its capacity factor, investments at preferable sites are incentivised.

In order to improve the quality of the initial spatial localisation of the generators, a warm start of the initial modelling year 2016 is performed. This is achieved by optimising the generation starting from the year 2010, following historic values for both capacities and energy output while simultaneously using the corresponding weather data to achieve the highest accuracy possible.

Detailed modelling of biomass, biogas, solar rooftop and solar ground-based, offshore and onshore wind, hydro storage and run of river generation, and also expansion planning, is based on historical weather years with weather conditions as of 2015 assumed for future years.

Investment cost and cost reduction rates from 2015 to 2025 are taken from [5] for wind and solar investments and from [6] for biomass. A moderate investment cost reduction rate of 5\% from 2025 to 2050 is assumed for all technologies except of wind-offshore with $15 \%$.

For computational reasons divestment decisions for existing generators are fixed at the end of the technical lifetime and all hourly resolved profile vectors are summed up to a yearly capacity factor of a generator. By doing so the number of variables is largely reduced.

2.1.2 Electricity demand: Spatial allocation of the yearly British electricity demand is performed according to the population data published on the third level of nomenclature of units for territorial statistic (NUTS). Based on the weighted areas on the NUTS-level, the assignment algorithm presented in [7] is performed to acquire the demand on the distribution grid substation level. National energy consumption values for each year were obtained from future energy scenarios (FES); however, transmission losses were excluded.

2.1.3 Spatial resolution and allocation: The approach includes a graph-based representation of the distribution grid, which bases on the previously published method for the German transmission grid [7], as well as an approximation of transport capacities within the transmission grid based on the dataset published in the context of the electricity ten year statement by National Grid.

The land-use based distribution of top down modelled potentials and profiles such as demand was achieved by overlaying the reference areas with Voronoi polygons based on the distribution and transmission grid substations. Next, the allocation of already existing, decommissioned, constructed, planned, or approved RES generators with known geographical positioning as well as the model-endogenously determined positions of greenfield and brownfield investment in RES generation are assigned to the nearest substation of either the transmission or distribution grid. The mathematical formulation of this approach can be found in [8].

Lastly, a dynamic assignment of demand and generation variables connected to the distribution grid is applied to the yearly changing transmission grid topology based on a shortest-path distribution over the distribution grid lines.

\subsection{Determination of the electricity wholesale market price effects}

The cost-minimisation model described in [9] is fed the most recent capacity predictions presented by NG in their FES to simulate wholesale electricity prices based on various generating capacity and fuel price predictions and then the dispatchable power from conventional thermal generators is calculated. The renewable power output is subtracted from electricity demand in order to create a net demand curve. Next, by stacking the thermal generators in a merit order, the generator supply function is formed. Lastly, wholesale prices of electricity for each time period are determined by the market clearing cost.

Table 1 Overview of main input sources for the renewable expansion model

\begin{tabular}{lllll}
\hline Type & Subtype & Capacity & National level & Regional level \\
\cline { 3 - 4 } & Nemand & NG demand data & NG FES 2016 & NG demand data \\
renewables & $\begin{array}{l}\text { wind } \\
\text { solar } \\
\text { run of river } \\
\text { biomass }\end{array}$ & NG FES 2016 & NG FES 2016 & $\begin{array}{l}\text { Eodel output } \\
\text { model output } \\
\text { ENTSO-E transparency platform } \\
\text { Elexon Data portal }\end{array}$ \\
\hline
\end{tabular}




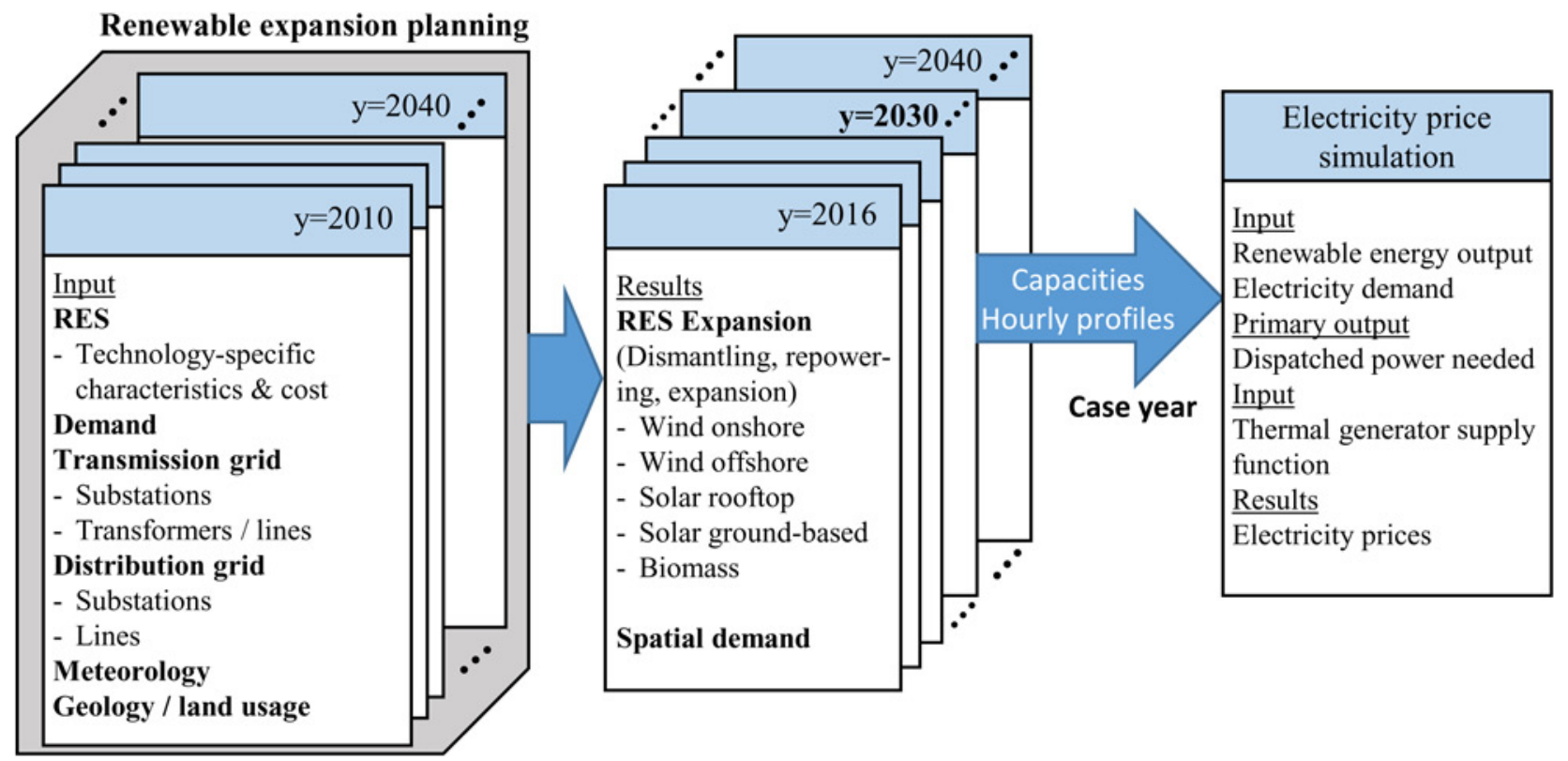

Fig. 2 Schematic overview of modelling approach

\subsection{Scenario generation and input data sources}

There are three different approaches for determining the best position for the installation of the renewable energy generation. The first one, MinCost, optimises the positioning just based on the predictions for the renewable capacity growth put forward by NG. The second one, GridConstraint, is also based on the NG capacity predictions but is constrained by the substation transmission capacity, which consists of transformer and line capacities of elements connected to the substation.

The last one, called EnergyConstraint, is bound by the constraints of the first and second approach, but also by the projected energy output of wind generation also provided by the NG's FES.

\section{Results}

\subsection{Cost of the transformation path and influence on the British electricity prices}

Fig. 3 displays three different simulations of electricity prices for the year 2030. The MinCost simulation displays the costs resulting from running the model according to the most optimal positioning of renewable resources, however, it does not account for grid constraints. These are accounted for the in the second graph, where a small increase in wholesale electricity prices can be

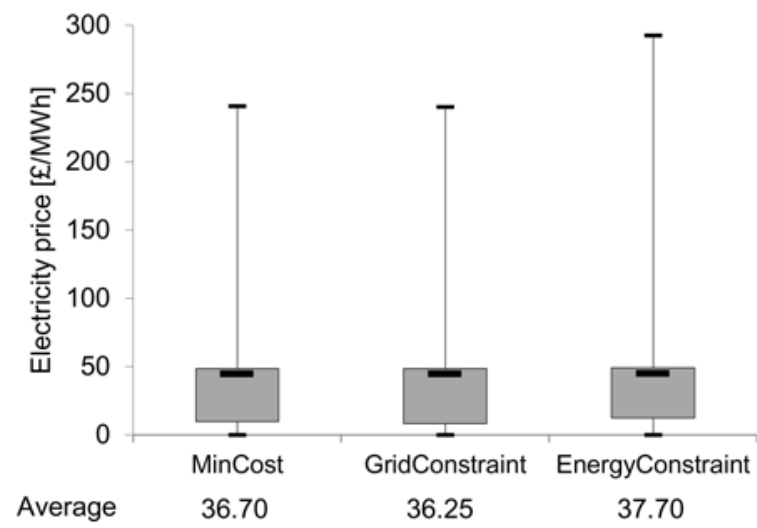

Fig. 3 Wholesale electricity prices for 2030 observed. The last graph takes into account optimal positioning of renewable resources, grid constraints, and the energy output constraint. Taking all three into account the biggest prices are observed. Although there are differences between the three scenarios, it is important to note they are very small and of little significance. While the fluctuation remains in the same reach for the scenarios MinCost and GridConstraint, it increases slightly in the EnergyConstraint scenario, along with the average price, which is still in the range of today's average wholesale market price of $40 \mathrm{f} / \mathrm{MWh}$. The increased spatial differentiation of the GridConstraint scenario leads ultimately to marginally lower prices than the MinCost scenario, due to the reduced concurrence of generation.

Fig. 4 displays the total discounted costs from 2016 to 2030 for all four NG FES when optimising RES expansion under consideration of the GridConstraint substation limitations. Since the biggest increase in renewable capacity installations is predicted in the gone green scenario, the overall cost increase corresponds to that.

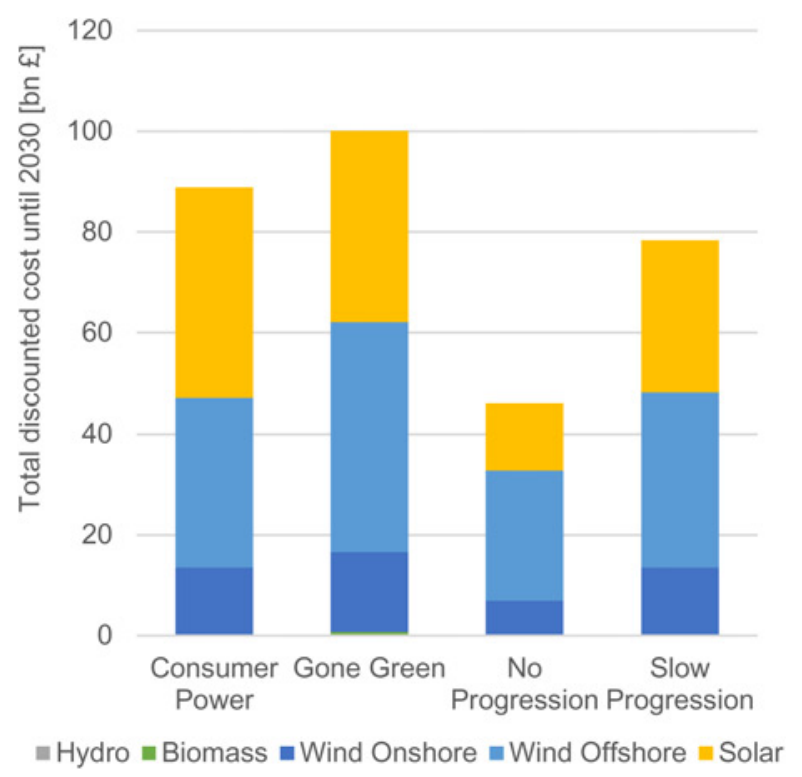

Fig. 4 Development of cost for renewable expansion in the GridConstraint scenario 
Further, as the cost of onshore wind continues to fall, the cost of its installation does not contribute significantly to the overall cost, which is mainly due to most expensive renewable generation, offshore wind. Offshore wind costs are decreasing, however, for the time being and in terms of future predictions, it maintains the highest cost, [5]. Widespread solar installations see solar reach a capacity of over $30 \mathrm{GW}$ in both Gone Green and Consumer Power. This contributes significantly to the total cost, observed by making a comparison with the no progression scenario, where solar capacity in 2030 is only $14.7 \mathrm{GW}$, and because of that, keeps the total scenario cost much lower compared to the other three.

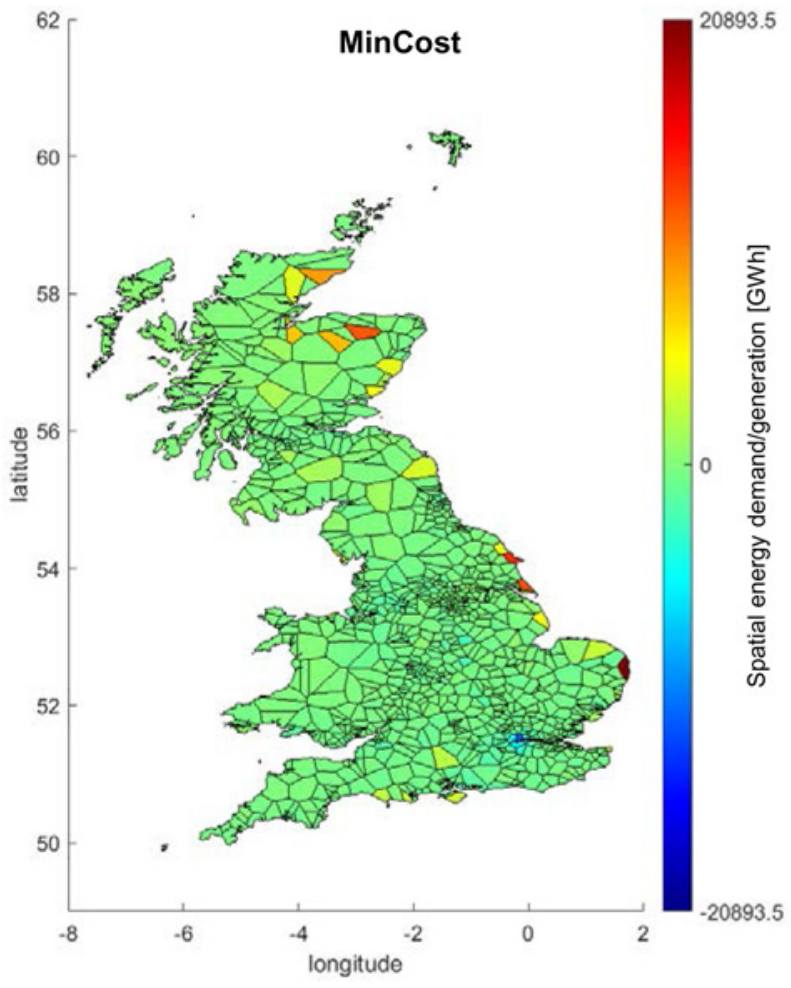

Fig. 5 Yearly energy balance per subregion

\subsection{Regional results}

Fig. 5 shows the energy balance of demand (negative values) and RES generation (positive values) in case of the MinCost approach for each assignment area in Britain. While the demand centres can expectably be found in areas with dense population, the areas with a high yearly net generation, which is mainly from wind onshore, land connection of offshore wind, and biomass generation, are found at the eastern coast as well as in the Scottish Highlands. In order to evaluate the regional effect of the three variants of the renewable generation expansion, the regional results in regard to the total installed capacity of RES can be found in Fig. 6. Fig. 6 shows capacity from landside connection of wind offshore in another colourset for improved readability. The inclusion of additional constraints in the GridConstraint and EnergyConstraint approach led to a reduction in the maximum capacity per region. This resulted in a shift in installed capacities from the north to more southern regions.

\section{Conclusions and outlook}

The paper analysed and presented the impact of increasing renewable generation throughout Great Britain for various regions, the cost that would incur with renewable installations, and the overall impact on wholesale electricity prices. The approach used in this paper expands from existing capacity projections by further determining the spatial RES expansion including deconstruction and repowering measures. The results demonstrate that the optimal locations for the installation of the future renewable capacity build vary significantly when considering the grid constraints. Subsequently, it was analysed how the electricity market would respond to such changes and what would the necessary costs be. The price simulation reveals that optimal locations, neglecting output and grid constraints, do not lead to significantly lower prices. This can explicitly be seen when considering the additional cost due to the additional grid expansion measures required as well as the total renewable expansion cost of the conversion path until 2030. Comparing today's expansion, which focuses on the North of the country, with the optimal spatial RES distribution determined in this paper reveals, that the existing expansion does not lead to ideal results when considering factors beyond just the amount of energy generated. Lastly, since the paper's main focus was optimal installation of renewable generation, the regional impact of the planned increase of gas-fired and nuclear generation, such as Hinkley Point $\mathrm{C}$, on the available capacity of the grid in areas
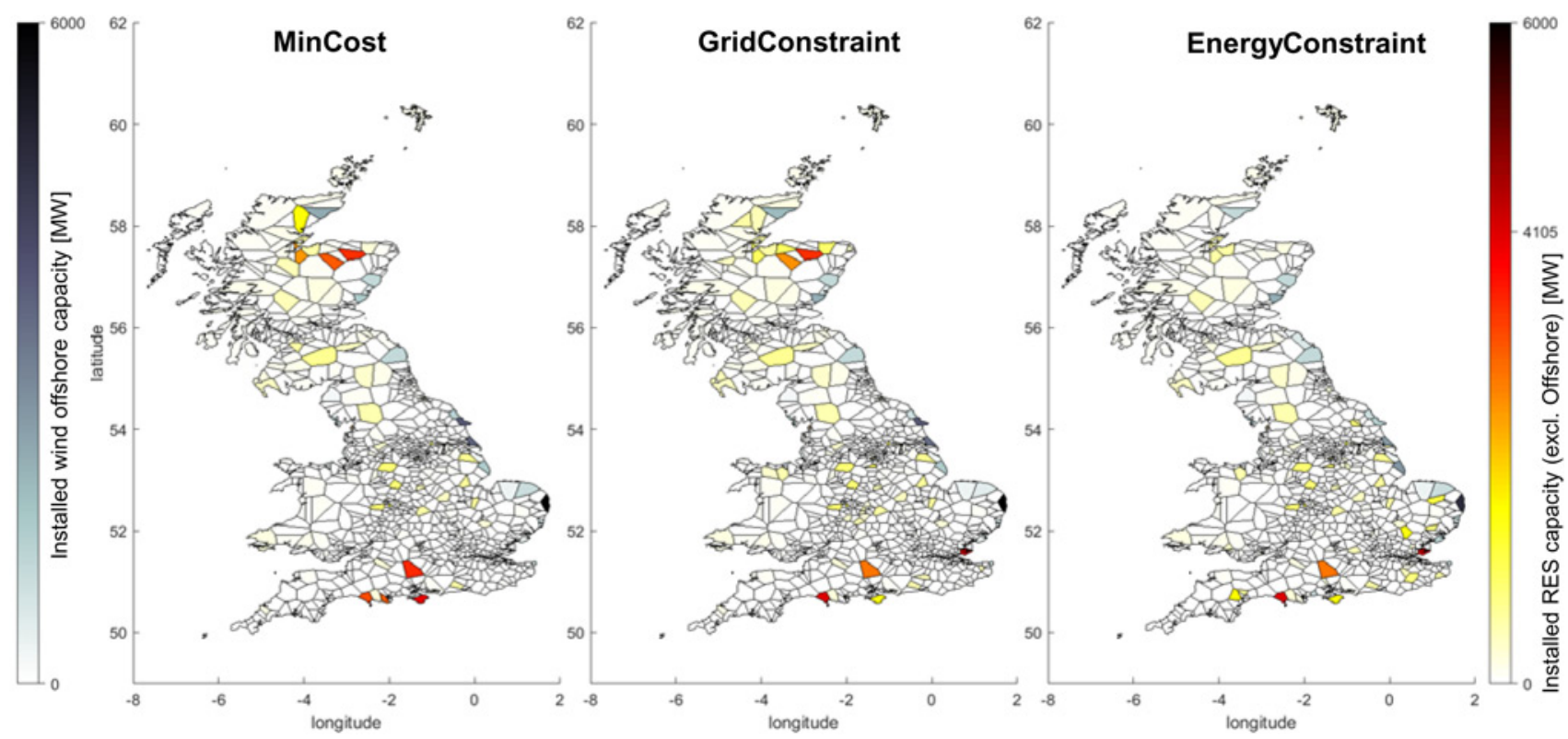

Fig. 6 Spatial distribution of installed capacities in the year 2030 
with concentration of thermal and nuclear power plants, was not included in this approach and could further improve the results quality.

\section{References}

1 Pfenninger, S., Keirstead, J.: 'Renewables, nuclear, or fossil fuels? Scenarios for Great Britain's power system considering costs, emissions and energy security', Appl. Energy, 2015, 152, pp. 83-93

2 Watson, J. J. W., Hudson, M. D.: 'Regional Scale wind farm and solar farm suitability assessment using GIS-assisted multi-criteria evaluation', Landscape Urban Plann., 2015, 138, pp. 20-31

3 National Grid: 'UK future energy scenarios', 2016
4 RenewableUK: 'UK wind energy database', 2017. Available at http://www. renewableuk.com/en/renewable-energy/wind-energy/uk-wind-energy-database/ index.cfm

5 IRENA: 'The power to change: solar and wind cost reduction potential to 2025 ', 2016

6 IRENA: 'Biomass for power generation', Irena Working Paper, vol. 1, 2012

7 Slednev, V., Ruppert, M., Bertsch, V., et al.: 'Regionalizing input data for generation and transmission expansion planning models', in Bertsch, V., Fichtner, W., Heuveline, V., Leibfried, T. (Eds.): 'Adv. Energy Syst. Optim. (in press)' (Trends in Mathematics, Birkhäuser, Cham, 2017)

8 Slednev, V., Bertsch, V., Ruppert, M., et al.: 'Highly resolved optimal renewable allocation planning in power systems under consideration of dynamic grid topology', MPRA Paper No. 79706, 2017, Available at https://mpra.ub.unimuenchen.de/79706/

9 Lupo, S., Kiprakis, A. E.: 'The impact of renewable energy resources on the electricity prices of the United Kingdom'. Proc. 13th Int. Conf. on the European Energy Market, Porto, 2016 